\title{
Long Term Sustained Growth of Insulin Producing Cells Using Agarose-Chitosan Coated Silver Nanocomposites
}

\author{
Kumar $\mathbf{N}^{1}$, Padhi $\mathrm{A}^{2}$, Sonawane $\mathrm{A}^{3}$ and Ganguly $\mathrm{A}^{\mathbf{1}^{*}}$ \\ ${ }^{1}$ Department of Biological Sciences, BITS-Pilani, KK Birla Goa Campus, Goa, India \\ ${ }^{2}$ School of Biotechnology, KIIT University, Bhubaneswar, India \\ ${ }^{3}$ Center for Biosciences and Biomedical Engineering, IIT, Indore, India
}

"Correspondence: Anasuya Ganguly, Department of Biological Sciences, BITS-Pilani, KK Birla Goa Campus, Goa, India

Received on 27 September 2020; Accepted on 7 January 2021; Published on 01 February 2021

Copyright $\odot 2021$ Kumar N, et al. This is an open access article and is distributed under the Creative Commons Attribution License, which permits unrestricted use, distribution, and reproduction in any medium, provided the original work is properly cited.

\begin{abstract}
Diabetes is a group of diseases characterized by high levels of blood sugar for an extended period. Despite newer and effective therapy, current treatment is riddled with fundamental challenges. To overcome the adverse effects of existing drugs, regenerative medicine has emerged as an essential treatment, for which tissue engineering may serve as a foundation for the repair of pancreatic cells secreting insulin. Different polymeric scaffolds have been explored for pancreatic tissue engineering. In the current study, a continuation of our preceding work we attempt to test the role of previously synthesized agarose-chitosan coated silver nanocomposite scaffold (AG-CHNp) for the long-term growth of pancreatic cells. Pancreatic cells were isolated from $B A L B / c$ mice and were characterized by dithizone (DTZ) staining, real time polymerase chain reaction (RT-PCR), western blotting, and flow cytometry for characteristic pancreatic markers. The isolated population of cells was grown on scaffolds and its effectiveness towards insulin secretion was studied. The isolated population was found to be positive for glucagon, PDX-1 and Pax-4, while a 200-fold change transcript level of insulin was observed. The cells upon seeding on the scaffolds exhibited sustained growth and insulin secretion as confirmed by western blotting. Overall, the study demonstrates the suitability and application of AG-CHNp for pancreatic tissue engineering.
\end{abstract}

Keywords: agarose, chitosan, scaffolds, islets, insulin, diabetes, tissue engineering, nanocomposites

\begin{abstract}
Abbreviations: DM: diabetes mellitus; T1D: type 1 diabetes; T2D: type 2 diabetes; IDDM: insulin dependent diabetes mellitus; ECM: extracellular matrix; AG-CHNp: agarose-chitosan coated silver nanocomposite scaffold; HBSS: hank's balanced salt solution; FBS: fetal bovine serum; DTZ: dithizone; PBS: phosphate buffered saline; DTT: dithiothreitol; EDTA: ethylenediaminetetraacetic acid; PMSF: phenylmethylsulfonyl fluoride; PVDF: polyvinylidene difluoride; PBST: phosphate buffered saline with 1\% tween 20; HRP: horseradish peroxidase; BSA: bovine serum
\end{abstract}


albumin; FITC: fluorescein isothiocyanate; FSC: forward scatter; SSC: side scatter; 2D: two dimensional; MTT: (3(4,5-dimethylthiazol-2-yl)-2,5-diphenyltetrazolium bromide); DMSO: dimethyl sulfoxide; DAPI: 4',6-diamidino-2phenylindole; 3D: three dimensional; RT-PCR: real time polymerase chain reaction; KRB Buffer: Krebs-Ringer bicarbonate buffer

\section{Introduction}

Diabetes mellitus (DM) is a metabolic disorder that is characterized by a chronic hyperglycaemic condition [1]. This condition primarily affects the islets of Langerhans situated in the pancreas and is mainly attributed to the defects in insulin secretion or action of insulin on cells or both [2]. Initially labeled as a disease of rich countries, diabetes has shown a tremendous increase in the past few years, even in middle-income nations. According to International Diabetes Federation Report (2019), a total of 463 million people across the world are currently suffering from diabetes [3]. It is also one of the leading causes of death around the world [4]. There are two main types of diabetes: type 1 diabetes (T1D) and type 2 diabetes (T2D). T1D, also known as insulin dependent diabetes mellitus (IDDM) is mainly caused by the inability of beta cells to secrete insulin, which in turn leads to an increase in the blood glucose level [5, 6]. It is an autoimmune disorder in which antibodies are generated against the pancreatic proteins. T2D on the other hand is a polygenic disorder where target tissues become insensitive to insulin and are generally associated with increased insulin resistance. T2D is also dependent on the lifestyle with obesity as a high-risk factor.

Currently, available treatment options include external insulin administration for T1D patients and the use of drugs to combat insulin resistance in T2D patients. After the discovery of insulin, there have been multiple modifications in the source and structure of insulin which have improved the state of diabetics around the world [7]. But still millions of people inject themselves with insulin multiple times a day, which not only is inconvenient but also has side effects and is not cost-effective. Various classes of drugs have been used for the management of diabetes either individually or in combination with each other. These include biguanides, sulfonylureas, megalitinides, thiazolidinediones, alphaglucosidase inhibitors, incretin-based therapies, dipeptidyl peptidase 1V inhibitor. All the types of drugs show some serious disadvantages ranging from nausea, vomiting, diarrhoea to immunogenic response, pancreatitis, and increased risk of myocardial infarction [8-11]. Further, T2D patients eventually resort to exogenous insulin administration in the long run.

With the disadvantages involved in the use of insulin, its analogues, and other pharmaceutical agents, attempts have been made to search for other more suitable options. These include whole pancreas transplantation, islet transplantation (human and porcine), and stem cell engineering to generate beta cells. First pancreatic transplantation was performed in 1966. Though initially pancreatic transplantation was not very successful, but with advances in surgical techniques and immunosuppression, there has been significant improvement in its efficiency [12]. In the year 2000, The "Edmonton protocol" for islet transplantation was a breakthrough in terms of providing long-term relief to diabetics. Edmonton protocol for transplantation requires, as many as 2-3 donor pancreases to procure sufficient islets for a single T1D patient becoming insulin independent [13]. However, there are complications associated with immune rejection and/or with chronic immunosuppressive treatment. 10 years hence, follow-up studies have also proven that multiple islet infusions are required per patient due to a loss of function of the cells over time [14, 15]. The shortage of suitable donors has also been a big obstacle thereby reducing its wide application.

The drawbacks associated with whole pancreas and islet transplantation have necessitated alternative treatment modalities and have paved the way for tissue engineering approaches. Tissue engineering is an amalgamation of multidisciplinary fields encompassing engineering, material science, and life sciences that aims to produce biologically viable substitutes for tissue and organ regeneration [16]. The biological substitutes thus can provide an alternative to bridge the ever-growing gap between demand and supply of organs for transplantation [17]. Scaffolds along with cells and bioactive molecules form the tissue engineering triad [18]. The correct combination of these 3 components aids in the development of a substitute for growing damaged tissues. Scaffolds are 3-dimensional structures which have a primary aim to impersonate the natural extracellular matrix (ECM) and provide the necessary 
structural and mechanical support to cells [17]. Previously, various polymers such as fibrin, silk, PEG, alginate etc. have been tested for the synthesis of scaffolds for their application in pancreatic tissue engineering [2].

Islets are small clusters of endocrine cells with a diameter of 50-200 $\mu \mathrm{m}$. Various mammalian islets that are used for research comprise of similar set of cells that differ in composition and cytoarchitecture. Various evidence corroborates that the functionality of the islets profoundly relies on their interaction with the ECM. Insulin secretion, islets survival, and proliferation have been shown to be regulated by the interactions with the ECM [19, 20]. Matrix interactions have also shown influence on islets development and $\beta$-cell differentiation [21, 22]. Therefore, it is crucial to construct a scaffold which can provide favorable environment for the islets to adhere, sustain and survive for longer period of time.

Agarose a naturally occurring polysaccharide has been used extensively in the field of tissue engineering because of its favorable properties like biodegradability, soft tissue like mechanical properties, rapid gelling capacity, etc. [23]. Agarose has been used as an encapsulating agent and blended with polymers (as scaffolds) for pancreatic tissue engineering [24, 25]. But its complete potential for islets engineering is yet to be explored. Chitosan, a positively charged polysaccharide is the most commonly used polymer in this field. With incredible properties like biocompatibility, biodegradability, anti-microbial activity, etc., chitosan has found a wide range of applications in tissue engineering [26, 27]. Silver nanoparticles are one of the most studied nanomaterials and have found application in various avenue of biomedicine such as wound healing, cancer therapy, anti-viral therapy, and tissue engineering [28, 29]. With properties like superior anti-microbial activity, reduced toxicity, due polymer coating, and better mechanical strength, silver nanoparticles have increasingly found application in engineering of bone, skin, etc. [30, 31].

In this study, we demonstrate the use of previously synthesized agarose-chitosan coated silver nanocomposite scaffolds (AG-CHNp) for pancreatic tissue engineering applications. These scaffolds have been prepared using the freeze-drying technique. Four different types of scaffolds containing different concentrations of chitosan were prepared (namely AG-CHNp1, AG-CHNp2, AG-CHNp3 and AG-CHNp4). The scaffolds were found to be biocompatible, biodegradable, hemocompatible, and showed optimum mechanical strength for soft tissue engineering [17]. In the current analysis, these unique scaffolds were tested for long term compatibility with mice pancreatic cells. Out of the four previously synthesized scaffolds, AG-CHNp4 gave the most promising results, hence for the present study, only AG-CHNp4 scaffolds were used.

\section{Materials and Methods}

\section{Isolation of pancreatic cells from mice}

Primary pancreatic cells were isolated using reported protocols $[32,33]$. Female BALB/c mice (age 6-8 weeks) were used for pancreas harvesting. Isolation was carried out under the approval of Institutional Animal Ethics Committee (registration number: 1577/PO/Re/S/2011/CPCSEA), Kalinga Institute of Industrial Research (KIIT), Bhubaneshwar, India. Briefly, mice were euthanized by diethyl ether. An incision was made in the abdominal area to expose the liver and intestine. The pancreas was then dissected and placed in a $50 \mathrm{ml}$ centrifuge tube containing collagenase XI (Sigma Aldrich, USA) prepared in Hank's balanced salt solution (HBSS). The centrifuge tube was then placed in $37^{\circ} \mathrm{C}$ for 15 min with occasional tapping to get cells in suspension. After the incubation, the tube was shaken vigorously to disrupt the pancreas completely. As the solution turned homogenous, the digestion was ceased using $1 \mathrm{mM}$ calcium chloride. Further, the solution was filtered through a 70-micron filter to remove any tissue debris. The solution was centrifuged at $1000 \mathrm{rpm}$ for $60 \mathrm{sec}$ at $4^{\circ} \mathrm{C}$ and the pellet after washing with $1 \mathrm{X}$ HBSS was resuspended in media containing RPMI 1640-GlutaMAX, 10\% FBS and antibiotics (Thermo Fisher Scientific, USA).

\section{Dithizone staining}

Dithizone (DTZ) staining was performed for mice pancreatic primary cells as described previously by Akira et al. [34]. $50 \mathrm{mg}$ DTZ (Sigma Aldrich) was dissolved in $5 \mathrm{ml}$ dimethyl sulfoxide (DMSO) (Thomas Baker Pvt. Ltd.) and 
filter sterilized to prepare the stock solution. Working solution was prepared by adding $10 \mu \mathrm{DTZ}$ stock solution to 1 $\mathrm{ml}$ media. The cells were washed with $1 \mathrm{X}$ phosphate buffered saline (PBS) and working solution was added to the cells and incubated for $15 \mathrm{~min}$. Post incubation, media was removed, and cells were washed with $1 \mathrm{X}$ HBSS. The stained clusters were examined under the microscope.

\section{Real-time polymerase chain reaction}

Total RNA was isolated from mice pancreatic cells using Trizol reagent as per manufacturer's instructions (Takara, Japan). Following extraction, $1 \mu \mathrm{g}$ of RNA was reverse transcribed to cDNA using RevertAid First strand cDNA synthesis kit (Thermo Fisher Scientific, USA). The synthesized cDNA was used as template to perform real-time polymerase chain reaction (RT-PCR) to check the expression of pancreatic markers (glucagon, pancreatic amylase, insulin and PDX-1) using gene specific primers (Table 1). $1 \mu 1$ of cDNA was applied in a total reaction mixture of 10 $\mu l$ containing $5 \mu \mathrm{l}$ of SYBR@ Green PCR master mix (Applied Biosystems, USA), $1 \mu$ l each of forward and reverse primers and $2 \mu \mathrm{l}$ of nuclease free water. This reaction was carried out for 40 cycles in Insta Q96 (Hi-Media, India). The mRNA levels were normalized to the transcript levels of GAPDH and absolute fold change was calculated.

\begin{tabular}{|l|l|l|}
\hline Gene & Forward primer & Reverse primer \\
\hline GAPDH & GTGAAGGTCGGTGTGAACGG & GATGCAGGGATGATGTTCTG \\
\hline Glucagon & CACTACCAGGGCACATTCACC & ACCAGCCACGCAATGAATTCCTT \\
\hline PDX-1 & GCGGTGGGGGCGAAGAGCCGGA & GACGCCTGGGGGCACGGCACCT \\
\hline Insulin & TTCTTCTACACACCCAAGAC & CTAGTTGCAGTAGTTCTCCA \\
\hline Pancreatic amylase 2 & TGGCGTCAAATCAGGAACATGG & GGCTGACAAAGCCCAGTCATCA \\
\hline
\end{tabular}

Table 1: Primer sequences for q-PCR.

\section{Western blotting}

Western blot analysis was performed to study the expression of various pancreatic cell markers. Cells isolated from mice pancreas were lysed by adding $100 \mu \mathrm{l}$ lysis buffer (1 M Tris, $2 \mathrm{M} \mathrm{Nacl,} 0.1$ M EDTA, $100 \mathrm{mM}$ DTT, $1 \%$ Triton $\mathrm{X} 100, \mathrm{Na}_{3} \mathrm{VO}_{4} \cdot 2 \mathrm{H}_{2} \mathrm{O}, 10 \%$ glycerol and PMSF) and stored in $-80^{\circ} \mathrm{C}$ overnight. The lysates were thawed on ice, briefly vortexed for $30 \mathrm{sec}$ thrice and centrifuged at 13,000 g for $30 \mathrm{~min}$. After estimation with Bradford assay (Bio-Rad, USA), $30 \mathrm{ug}$ of protein was resolved using 12\% SDS-PAGE and transferred onto polyvinylidene difluoride (PVDF) membrane (Bio-Rad, USA) for $70 \mathrm{~min}$ at $80 \mathrm{~V}$. The membranes were blocked with 5\% skimmed milk (Hi-Media, India) for $2 \mathrm{~h}$ at room temperature. After blocking, the membranes were incubated overnight with primary antibody at $4^{\circ} \mathrm{C}$. Post-incubation, the membranes were washed thrice with $1 \mathrm{X}$ PBS containing $1 \%$ Tween 20 (PBST). The washed membrane was probed with horseradish peroxidase (HRP) tagged secondary antibody for $2 \mathrm{~h}$ at room temperature. Finally, the membranes, after washing with $1 \mathrm{X}$ PBST, were developed on X-ray film using chemiluminescent solvents (Millipore, USA Catalogue: WBKLS00). Primary antibodies used: PDX-1 (Santa Cruz Biotechnology sc-390792; 1:2500), Pax-4 (Santa Cruz Biotechnology sc-98941; 1:2500), glucagon (Santa Cruz Biotechnology sc-514592; 1:2500), insulin (Santa Cruz Biotechnology sc-98941; 1:2500), $\beta$-actin (Cell Signaling 4970; 1:5000). Secondary antibodies used: Goat anti-rabbit IgG HRP conjugated (Merck Millipore, USA 6.2114E14; 1:7500) and anti-mouse IgG HRP linked antibody (Cell Signaling 7076; 1:5000).

\section{Flow cytometry analysis}

Cells were washed with incubation buffer (0.5\% BSA in 1X PBS) and fixed using $2 \%$ formaldehyde for 15 min. This was followed by permeabilization with $0.1 \%$ Triton X in $1 \mathrm{X}$ PBS for $30 \mathrm{~min}$. Cells were then incubated with primary antibody for PDX-1 and insulin for $1 \mathrm{~h}$ at room temperature. Cells were further incubated with fluorescein isothiocyanate (FITC) tagged secondary antibody for $30 \mathrm{~min}$ at room temperature. Stained cells were subjected to flow cytometric analysis using BD FACS Canto II cytometer and analysed using FACS Diva software. Multiple gating strategies were used to gate the correct events. Forward scatter (FSC) area $v s$ height measurement was done to remove clumps for single cell analysis, single cells falling along a diagonal were chosen for further analysis. FSC and side scatter (SSC) were used to gate viable and single cells events. Gating was done to exclude debris and dead cells from 
analysis by categorizing low FSC events as debris, and events with low FSC and high SSC as dead cells. A compact cell population was thus gated based on size and granularity of the registered events on the scatter plot. Gated PDX-1 and insulin positive cells were determined as proportionate shift in population.

\section{MTT Assay}

AG-CHNp scaffolds have been previously studied for their biocompatibility against various cell lines [17]. To check for biocompatibility with mice pancreatic primary cells, AG-CHNp4 scaffolds were placed in 24 well uncoated plates. The scaffolds were sterilized using ethanol gradient. This was followed by exposure to UV radiation for $20 \mathrm{~min}$. Complete media was added to the sterilized scaffolds and incubated for $4 \mathrm{~h}$ to promote equilibration of scaffolds for adequate gaseous exchange. Furthermore, the mice pancreatic primary cells were seeded onto the pre-sterilized scaffolds and on poly-lysine coated 24 well plate (which acts as $2 \mathrm{D}$ control) and incubated at $37^{\circ} \mathrm{C}$ with $5 \% \mathrm{CO}_{2}$ humidified chamber for a period of 40 days. Half media was changed every 5 days. On the day of the experiment, media was removed from the well and washed with PBS. MTT solution $(0.5 \mathrm{mg} / \mathrm{ml})$ was added for $3-4 \mathrm{~h}$. Following the incubation, DMSO was added and incubated for $20 \mathrm{~min}$. The purple color developed was measured at $570 \mathrm{~nm}$ to calculate cell viability.

\section{DAPI staining}

For microscopic analysis of the attachment and growth of mice pancreatic primary cells on AG-CHNp scaffolds, DAPI staining was performed. The cells were seeded on the AG-CHNp4 scaffolds and incubated at $37^{\circ} \mathrm{C}$ with $5 \% \mathrm{CO}_{2}$. One the day of the experiment, the scaffolds were washed with PBS and incubated overnight with $2.5 \%$ glutaraldehyde for fixation. Following day, scaffolds were rinsed with PBS and dried using ethanol gradient. DAPI mounting media (Sigma Aldrich, USA) was added to section of fixed scaffolds and incubated for $25 \mathrm{~min}$ in dark. The slides were observed using fluorescence microscope (excitation: $405 \mathrm{~nm}$ and emission: $450 \mathrm{~nm}$ ).

\section{Immunofluorescence}

Immunofluorescence was performed to confirm the presence of PDX-1, insulin and glucagon positive cells. The pancreatic primary cells were seeded onto the scaffolds for a period of 40 days. On the day of experiment, the media was removed, and the cells were fixed with $2.5 \%$ glutaraldehyde overnight. Post-fixation, sample dehydration was performed utilizing ethanol gradient, cleared with xylene and were paraffinized. For immunofluorescence, the embedded scaffolds were sectioned, deparaffinized and rehydrated. Furthermore, the sections were permeabilized using $0.1 \%$ triton $\mathrm{X}-100$ and blocked using $1 \% \mathrm{BSA}$ for $1 \mathrm{~h}$ at room temperature. The sections were incubated with primary antibody (PDX-1, insulin and glucagon) at $4^{\circ} \mathrm{C}$ overnight. Post-incubation, sections were washed with $1 \mathrm{X}$ PBS and probed with FITC tagged secondary antibody for $2 \mathrm{~h}$ at room temperature. After incubation, the sections were washed with $1 \mathrm{X}$ PBS, counterstained with DAPI mounting media for $15 \mathrm{~min}$ and observed using fluorescence microscope (Olympus BX63 automated fluorescence microscope).

\section{Qualitative estimation of insulin secretion}

For qualitative estimation of insulin secretion, western blotting was performed. Mice pancreatic primary cells were seeded on AG-CHNp4 scaffolds. The media which was added for the growth of cells (Spent media) was collected on various time points (day 5, 10, 20, 25, 30, 35, 40). The spent media was then centrifuged to remove any cellular debris. Freshly prepared complete media was used as control. The media was separated using $12 \%$ SDS PAGE gel and was transferred to a PVDF membrane. The membranes were blocked at room temperature using skimmed milk, followed by overnight incubation with the primary monoclonal anti-insulin antibody. Post-incubation, the membrane was washed with PBST and incubated with HRP tagged secondary antibody at room temperature. After the incubation, the membrane was washed with 1X PBST and chemiluminescent detection was carried out. 


\section{Glucose challenge test}

In order to further evaluate the functionality of pancreatic cells growing on the scaffolds, glucose stimulated insulin release was recorded [35]. Mice pancreatic primary cells were seeded on AG-CHNp4 scaffolds and grown for a period of 45 days. On the day of experiment, media was removed, and cells were washed with 1X PBS. Furthermore, the cells were incubated with Krebs-Ringer bicarbonate (KRB) buffer containing $3.3 \mathrm{mM}$ glucose for $1 \mathrm{~h}$. Post-incubation, the buffer was removed, and cells were washed with 1X PBS. Likewise, cells were then incubated with KRB buffer containing $17 \mathrm{mM}$ glucose for $1 \mathrm{~h}$ and the buffer was collected after glucose challenge, insulin secretion was qualitatively analysed by chemiluminescent detection using western blotting.

\section{Results}

\section{Characterization of cells isolated from mice}

\section{- Dithizone staining}

The isolated primary cells from mice were characterized using DTZ. DTZ is a sulphur containing compound which has specific affinity for zinc ions. Therefore, DTZ is used to stain zinc containing beta cells [36]. The figure (Figure 1) shows DTZ stained primary cells. The DTZ stained cells suggest the presence of beta-cell in the isolated cell preparation.

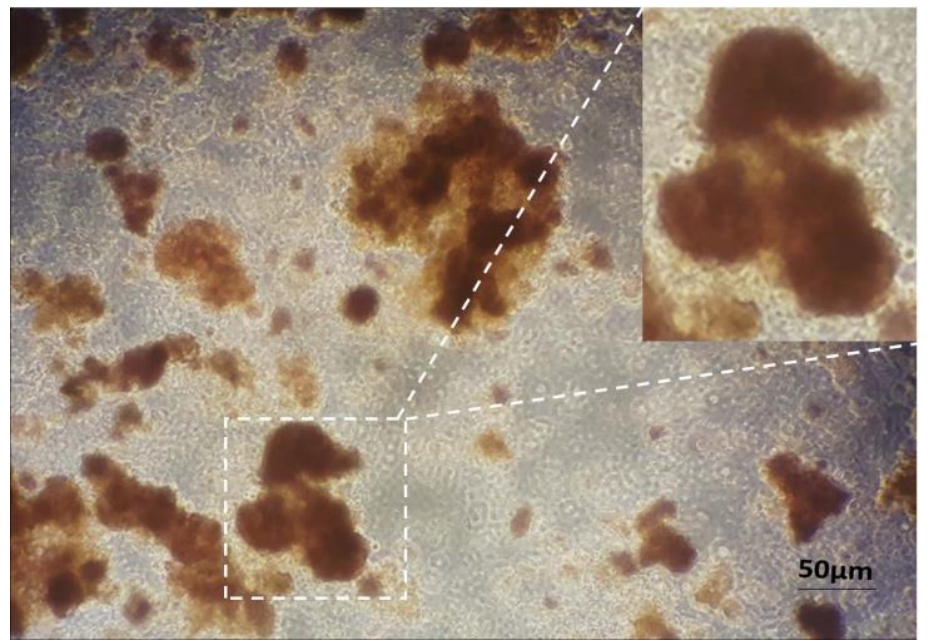

Figure 1: Characterization of mice primary pancreatic cells using dithizone staining. Dithizone staining was performed on isolated pancreatic cells which showed crimson, red-stained beta cells.

\section{- Gene expression profile}

To characterize the isolated cell preparation, a PCR based technique was employed to quantify the fold change in gene expression levels of signature pancreatic tissue markers. Isolated cells were grown on tissue culture plates for 4 days, harvested, total RNA isolated and c-DNA was synthesized. Expression levels of insulin, PDX-1, glucagon and amylase transcripts were determined using gene specific primers (Table 1). We found approximately 200 -fold change in mRNA transcript levels of insulin, one of the most important hormones secreted by pancreas participating in carbohydrate metabolism (Figure 2a). Similarly, higher levels of PDX-1, a transcription factor necessary for $\beta$-cell maturation was observed. However, we observed extremely low transcript levels of amylase relative to the reference gene. The transcript levels were normalized with housekeeping gene GAPDH and the fold changes were calculated. For primer specificity confirmation melting curve analysis was done for each set of primers. 


\section{- Protein expression profile of PDX-1, PAX-4 and insulin}

To confirm the mRNA level expression, western blotting was performed. The isolated proteins were transferred onto a PVDF membrane and expression levels for PDX-1, PAX-4 and insulin were studied using specific monoclonal antibodies. Western blotting analysis showed positive expression of PDX-1, PAX-4 and insulin (Figure 2b). However, the expression of insulin was considerably higher than PDX-1 and PAX-4. Furthermore, bands were also quantified using densitometric program in ImageJ and then were normalized with $\beta$-actin. SW1990, a pancreatic adenocarcinoma cell line was used as a positive control and THP-1, monocytic cell line was used as a negative control. Whole blots are supplied as supplementary figures (Supplementary Figure 1 and 2).

- Flow cytometry

The isolated mice pancreatic primary cell population was also characterized using flow cytometry to find out the percentage of PDX-1 and insulin positive cells. The cells were processed on the 4th day of culture. They were fixed and probed with specific monoclonal primary antibody followed by incubation with FITC tagged secondary antibody. Flow cytometric analysis of the cells revealed 22.3\% PDX-1 positive and 27.8\% insulin positive cells (Figure 2c) (Figure 2).

a

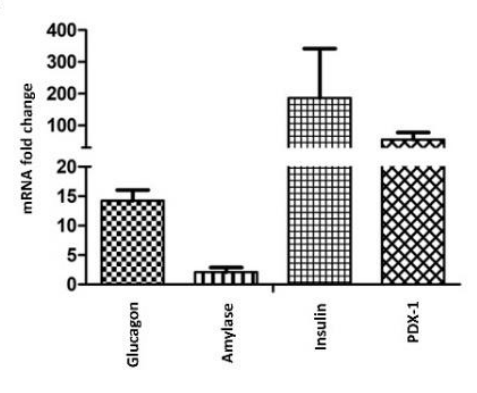

b

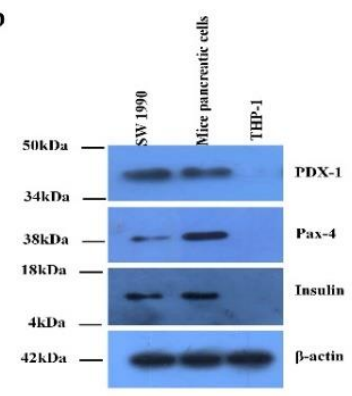

$\mathbf{c}$

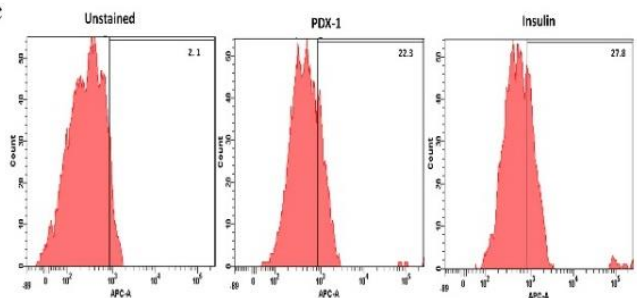

Figure 2: Characterization of mice primary pancreatic cells. (a) Relative expression of various pancreatic genes after four days of primary culture. Gene expression was relative to GAPDH. RT-PCR shows positive but varying expression of glucagon, amylase, insulin and PDX-1. The experiments were repeated in replicates $(n=3)$ and the graph is representative of the average of the same. (b) Western blot analysis detected the expression of Glucagon (18KDa), PDX-1 (34-50KDa), PAX-4 (38KDa) and Insulin (4-18KDa) in primary mice pancreatic cells (c) Flow cytometry analysis after four days of culture showed positive expression of PDX-1 (22.3\%) and Insulin (27.8\%).

\section{Characterization of the artificial construct with mice pancreatic primary cells}

\section{- Viability of cells in scaffold}

Long term viability of pancreatic cells on the 3D scaffold is an important component of tissue engineering. Cell cytotoxicity on the scaffolds was measured by MTT assay. Metabolically active cells on the scaffolds convert MTT salt to formazan crystals which gets solubilized in DMSO and gives a purple colour. This colour is quantitated at 570 $\mathrm{nm}$ using a spectrophotometer. In the current study, the proliferative capacity of the pancreatic cells was studied for a span of 40 days (Figure 3a). Cells growing on tissue culture plates were used as 2D control. Initially till day 10, 2D control showed better cell proliferation but with reduced surface area available for further propagation, there was reduction in cell number. On the other hand, AG-CHNp4 scaffold provide ideal growth environment for scaffolds and more surface area owing to the 3-dimensional morphology, showed increased growth with each time point. At the end 
of 40 days, AG-CHNp4 scaffold showed sustained growth of cells as compared to 2D control where the growth reduced with time.

- Microscopic analysis of the construct

To study the attachment and proliferation capacity of the pancreatic cells on the AG-CHNp4 scaffolds, DAPI staining was performed. DAPI staining showed rounded nucleus of the cells and this was observed in the scaffolds after $48 \mathrm{~h}$ of culture (Figure 3b). Presence of an intact nuclei indicates that the cells are viable inside the scaffolds (Figure 3).

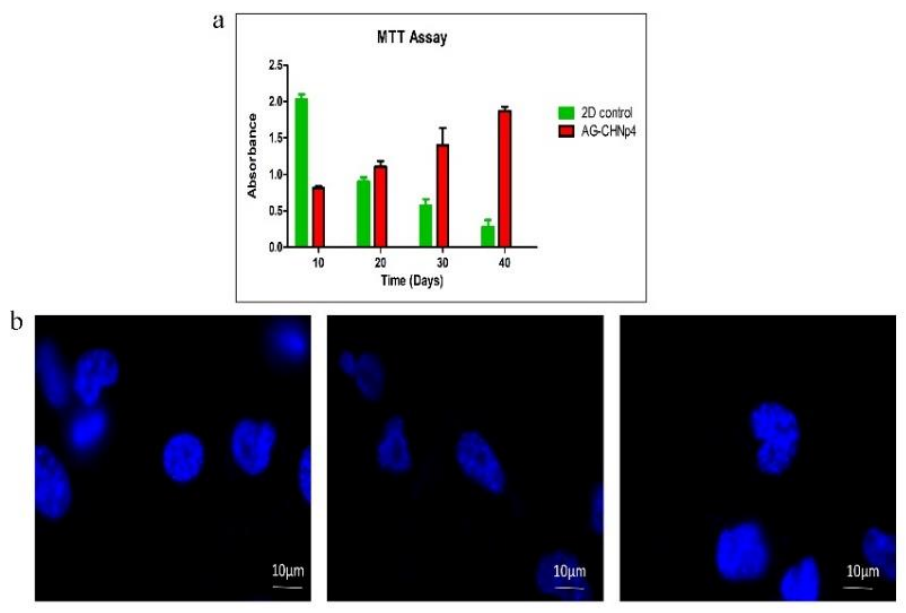

Figure 3: Cell viability and proliferation was studied with MTT assay. (a) Mice pancreatic primary cells (Passage 0) growing on AG-CHNp4 scaffolds showed prolonged and sustained growth even on day 40 as compared to $2 \mathrm{D}$ control where radical cell death was visible upon reaching confluency. The experiments were repeated in replicates $(n=3)$ and the graph is representative of the average of the same. (b) DAPI staining showed distinct morphology and shape of the cells on day 2 of culture.

- Expression of pancreatic markers in cells growing on the scaffold

To comprehend whether the pancreatic cells growing on the scaffolds contains insulin producing $\beta$-cells, we explored the level of expression of various pancreatic markers such as PDX-1, insulin and glucagon. PDX-1 is a transcription factor necessary for pancreatic development and $\beta$-cell maturation. Developing $\beta$-cells produce PDX-1, whose expression seemingly favors the production of insulin secreting $\beta$-cells. Thus, increased PDX-1 expression in isolated cells indicates increased survival of $\beta$-cells. On the other hand, glucagon and insulin are peptide hormones secreted by alpha and beta-cells of pancreatic islets respectively. These 2 hormones work antagonistically to each other thereby, maintaining glucose metabolism in the body. To show the expression of above-mentioned markers in the pancreatic cells seeded on AG-CHNp4 scaffolds, we performed the immunostaining for PDX-1, insulin and glucagon using marker specific monoclonal antibody. 30 days post-incubation, the scaffolds were paraffinized, and these sections were cut using microtome. The thin sections were then deparaffinized and rehydrated, stained for specific markers and observed under fluorescent microscope. As can be seen in the figure (Figure 4a), we observed significant levels of PDX-1 inside the nucleus of the cells. Localization of PDX-1 in the nucleus was confirmed by co-localizing DAPI (blue) and PDX-1 levels (green) which formed the cyan colour on merging. In the figure (Figure $4 \mathrm{~b}$ and $4 \mathrm{c}$ ), we observed substantial expression of insulin and glucagon. Cytoplasmic localisation of both insulin and glucagon was confirmed by merging DAPI (blue) and insulin (red) or glucagon (red) (Figure 4). Thus, the cells isolated from mice pancreas were surviving and maintaining their functionality even after 30 days in culture and were showing positive expression of PDX-1, insulin and glucagon.

\section{- Secretion of insulin inside the scaffolds}

Insulin secretion in human body is responsible for glucose metabolism. To check whether the tissue construct also secretes insulin, western blotting was performed (Figure 5a). Western blotting analysis showed positive expression of 
insulin in the spent media at different time points. Complete media used for growing pancreatic cells on the scaffold was used as the control. The bands were quantified using densitometric analysis and were normalized to day 5 time point as the complete media control did not show any expression of the insulin. Coomassie stained gel was used as the loading control. This result also confirms the results from immunofluorescence that the PDX-1 expressing cells were functionally active and were secreting insulin after 30 days of culture.

- Pancreatic cells growing on the scaffold secretes adequate levels of insulin

Regulation of insulin secretion under glucose stress is an integral segment of a functional pancreatic construct. Beta cells should be able to adjust to the levels of glucose and secrete adequate concentration of insulin. Insulin secretion upon glucose challenge was qualitatively studied using western blotting. After 45 days of culture, cells were incubated with KRB buffer containing different concentrations of glucose $(3.3 \mathrm{mM}$ and $17 \mathrm{mM})$. These buffers were run on a $12 \%$ SDS PAGE gel and were transferred onto PVDF membrane. Insulin was detected using chemiluminescent reagents. The figure (Figure 5b) shows differential levels of insulin in the incubated buffers. Low glucose showed lower intensity band as compared to high glucose concentration. This further confirms long term functionality of betacells growing on the scaffolds (Figure 5).

(a)

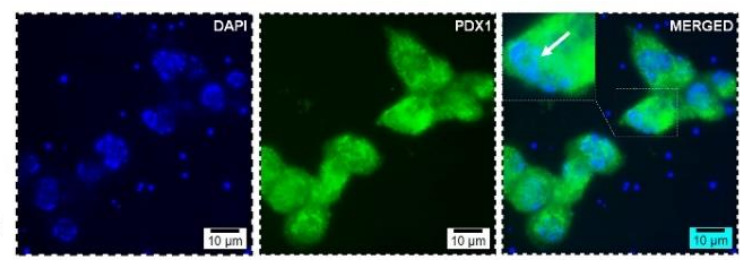

DAPI GLUCAGON MERGED
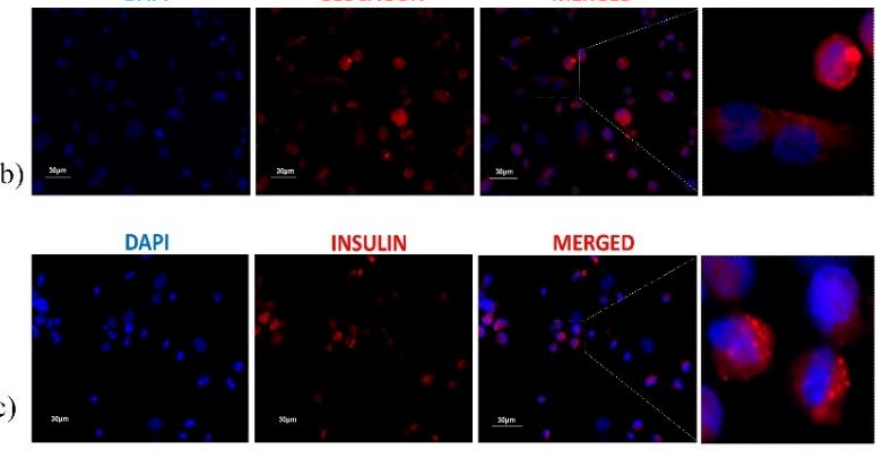

Figure 4: Microscopic analysis of mice pancreatic cells growing on AG-CHNp4 scaffold. Immunofluorescence staining showed localisation of (a) PDX-1 and positive expression of (b) glucagon and (c) insulin which confirms the presence of islets on Day 30 of pancreatic cell culture.
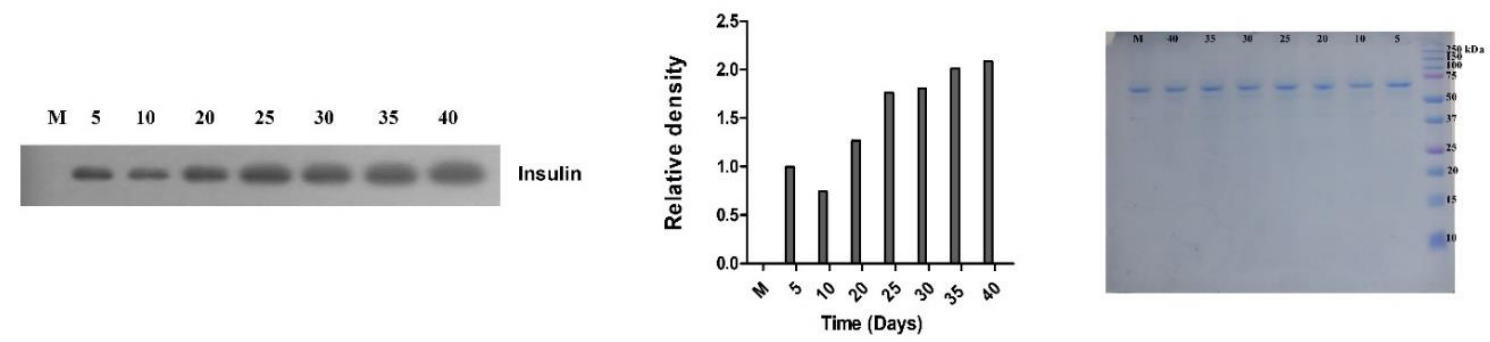

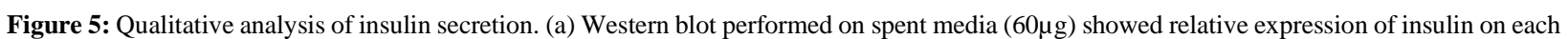
day from Day 5 to Day 40. M is the complete media which was used as control. Coomassie stained gel was used as loading control. (b) Qualitative analysis of insulin secretion after glucose challenge test showed its differential expression under different glucose concentrations. 


\section{Discussion}

Tissue engineering deals with developing renewable resource for organ replacement and regeneration [16, 37]. One of the important components in tissue engineering, scaffolds play an important part in fabrication of artificial tissue construct. As scaffolds act as the natural environment for cell survival, properties such as biocompatibility and biodegradability become paramount [18]. Various polymers have been tested for pancreatic tissue engineering, however since all the polymers are not well studied and documented in relation to engineering pancreas, the ideal polymeric scaffold has not yet been identified [2]. Moreover, it has been established that islets tend to lose viability post-isolation due to lack of ECM [38]. Therefore, appropriate choice of polymer for scaffold design is extremely important. Another setback with pancreatic tissue engineering is the complexity of the organ itself. Pancreas consists of multiple types of cells and every cell type has an important role to play in the overall functioning of the organ [39].

In the current study, a nanocomposite scaffold made up of agarose and chitosan coated silver nanoparticles was used for application in pancreatic tissue engineering (Figure 6). AG-CHNp scaffold synthesized using the freeze-drying technique, has shown favorable properties such as biocompatibility, biodegradability, good swelling ratio and suitable mechanical strength as previously reported by our group [17]. In preceding reports, agarose-based scaffolds have been used for tissue engineering applications such as for cartilage, liver and implantation surgery [40, 41]. For pancreatic tissue engineering, agarose has been primarily studied as an encapsulating agent. Few studies have been conducted using agarose as a 3D scaffold. One group recently showed the effect of co-culturing bone marrow derived mesenchymal stem cells and human islets on agarose gel which proved application of agarose in pancreatic tissue engineering [42]. But as agarose gel does not support growth of cells, agarose alone might not fit the criteria.

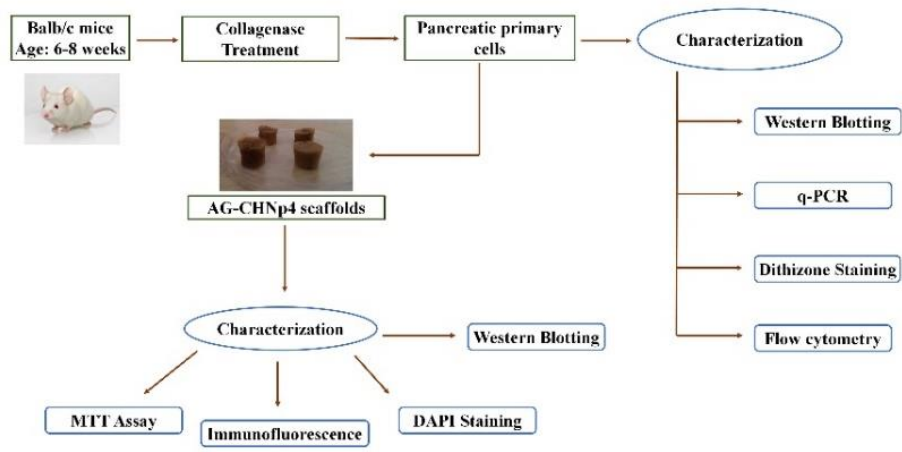

Figure 6: Schematic representation showing the application of AG-CHNp4 scaffolds for pancreatic tissue engineering. Mice primary pancreatic cells were isolated from BALB/c mice and were characterized using western blotting, RT-PCR, dithizone staining and flow cytometry. These cells were cultured on AG-CHNp4 scaffold and the cell construct was characterized by various molecular techniques viz MTT assay, DAPI staining, immunofluorescence and western Blotting.

The present report studies the collective effect of all the pancreatic cell types onto the AG-CHNp4 scaffold. The pancreatic primary cells were isolated from BALB/c mice and characterized using techniques such as DTZ staining, RT-PCR, western blotting and flow cytometry. DTZ staining confirmed the presence of islets by staining them crimson red. RT-PCR showed variable but positive expression for various pancreatic markers (glucagon, insulin, amylase and PDX-1) suggesting mixed population of cells. This result was further confirmed by western blotting which showed positive expression for PDX-1, PAX-4 and insulin. The western blotting result is concurrent to the fact that insulin is a secreted protein and will show higher levels in comparison to PDX-1 and PAX-4 which are transcription factors associated with differentiated beta cells. Further to investigate the role of AG-CHNp4 scaffold as an artificial ECM for pancreatic tissue engineering, the isolated cells were grown on the scaffold and characterized at various time points. The viability of the cells was established with MTT assay which showed good attachment and proliferation of cells for a period of 40 days in comparison to $2 \mathrm{D}$ culture system where the cell number reduced drastically after attaining confluency. This experiment highlights the role of AG-CHNp4 scaffold for long term growth of pancreatic cells. To further confirm the presence of beta-cells growing on the scaffold, immunofluorescence was performed for PDX-1, insulin and glucagon. PDX-1 is a transcription factor associated with beta cell maturation and pancreatic development. 
Insulin and glucagon are important hormones for glucose metabolism. After 30 days of culture, pancreatic cells showed positive PDX-1, insulin and glucagon expression which shows the viability of beta cells in AG-CHNp4 scaffolds. To corroborate the findings at translational level, western blotting was performed on the spent media from the 3D culture system. Western blotting analysis confirmed insulin secretion at all the time points starting from day 5 to day 40 which confirmed sustainable growth of beta-cells for long period of time in vitro. Finally, functionality of beta cells was also confirmed by glucose challenge assay which showed differential intensity peaks for high and low glucose concentrations.

In conclusion, to our best knowledge this is the first report suggesting the application of nanoparticle-based composite for studying growth and survival of pancreatic cells for a prolonged period. Agarose-chitosan silver nanoparticle composite shows pronounced compatibility with respect to mice pancreatic primary cells in vitro which was successfully characterized at the transcriptional and translational level. Further analysis needs to be performed at the in vivo level to further confirm our findings. With these current results, AG-CHNp4 scaffold looks very promising in the field of pancreatic tissue engineering.

\section{Author Disclosure Statement}

The authors declare no competing financial interests.

\section{Funding}

This work is supported by the Department of Science and Technology/SERB (SR/FT/LS-137/2011). NK would like to acknowledge the Indian Council of Medical Research (BMS/FW/SCR/2015-20430/MAR-2015/06/GA/PVT) for her senior research fellowship.

\section{Supplementary Material}

The supplementary material for this article can be found online at: https://seriesscience.com/wpcontent/uploads/2021/02/Supplementary-Material.pdf

\section{References}

1. Alam U, Asghar O, Azmi S, et al. General aspects of diabetes mellitus. Handb Clin Neurol. 2014;126:21122.

2. Kumar N, Joisher H, Ganguly A. Polymeric scaffolds for pancreatic tissue engineering: A review. Rev Diabet Stud. 2018;14(4):334-53.

3. International Diabetes Federation. IDF Diabetes Atlas. 7th ed., Brussels, Belgium, 2015.

4. World Health Organization. Global report on diabetes. 2016.

5. van Belle TL, Coppieters KT, von Herrath MG. Type 1 diabetes: etiology, immunology, and therapeutic strategies. Physiol Rev. 2011;91(1):79-118.

6. Butalia S, Kaplan G, Khokhar B, et al. Environmental risk factors and type 1 diabetes: Past, present, and future. Can J Diabetes. 2016;40(6):586-93.

7. Greene JA, Kevin RR. Why there is no generic insulin? Historical origins of a modern problem. N Engl J Med. 2015;372(12):1171-175.

8. Shorr RI, Ray WA, Daugherty JR, et al. Individual sulfonylureas and serious hypoglycemia in older people. J Am Geriatr Soc. 1996;44(7):751-55. 
9. Olokoba AB, Obateru OA, Olokoba LB. Type 2 diabetes mellitus: A review of current trends. Oman Med J. 2012;27(4):269-73.

10. Nissen SE, Wolski K. Effect of rosiglitazone on the risk of myocardial infarction and death from cardiovascular causes. N Engl J Med. 2007;356(24):2457-471.

11. Lovshin JA, Drucker DJ. Incretin-based therapies for type 2 diabetes mellitus. Nat Rev Endocrinol. 2009;5(5):262-69.

12. Niclauss N, Meier R, Bédat B, Berishvili E, Berney T. Beta-cell replacement: Pancreas and islet cell transplantation. Endocr Dev. 2016;31:146-62.

13. Shapiro AM, Lakey JR, Ryan EA, et al. Islet transplantation in seven patients with type 1 diabetes mellitus using a glucocorticoid-free immunosuppressive regimen. N Engl J Med. 2000;343(4):230-38.

14. Ryan EA, Paty BW, Senior PA, et al. Five-year follow-up after clinical islet transplantation. 2005;54(7):2060-69.

15. Shapiro AM, Ricordi C, Hering BJ, et al. International trial of the Edmonton protocol for islet transplantation. N Engl J Med. 2006;355(13):1318-330.

16. Sengupta D, Waldman SD, Li S. From in vitro to in situ tissue engineering. Ann Biomed Eng. 2014;42(7):1537-545.

17. Kumar N, Desagani D, Chandran G, et al. Biocompatible agarose-chitosan coated silver nanoparticle composite for soft tissue engineering applications. Artif Cells Nanomed Biotechnol. 2017;46(3):637-49.

18. Asghari F, Samiei M, Adibkia K, et al. Biodegradable and biocompatible polymers for tissue engineering application: A review. Artif Cells Nanomed Biotechnol. 2017;45(2):185-92.

19. Pinkse GGM, Bouwman WP, Jiawan-Lalai R, et al. Integrin signaling via RGD peptides and anti-beta1 antibodies confers resistance to apoptosis in islets of Langerhans. Diabetes. 2006;55(2):312-17.

20. Beattie GM, Montgomery AM, Lopez AD, et al. A novel approach to increase human islet cell mass while preserving beta-cell function. Diabetes. 2002;51(12):3435-439.

21. Beattie GM, Cirulli V, Lopez AD, et al. Ex vivo expansion of human pancreatic endocrine cells. J Clin Endocrinol Metab. 1997;82(6):1852-856.

22. Kaido T, Perez B, Yebra M, et al. $\alpha$ v-Integrin utilization in human $\beta$-cell adhesion, spreading, and Motility. 2004;279(17):17731-7737.

23. Kohane DS, Langer R. Polymeric biomaterials in tissue engineering. Pediatr Res. 2008;63(5):487-91.

24. Gazda LS, Vinerean HV, Laramore MA, et al. Pravastatin improves glucose regulation and biocompatibility of agarose encapsulated porcine islets following transplantation into pancreatectomized dogs. J Diabetes Res. 2014;2014;405362.

25. Hilderink J, Spijker S, Carlotti F, et al. Controlled aggregation of primary human pancreatic islet cells leads to glucose-responsive pseudoislets comparable to native islets. J Cell Mol Med. 2015;19(8):1836-846.

26. Cao Z, Gilbert RJ, He W. Simple Agarose-chitosan gel composite system for enhanced neuronal growth in three dimensions. Biomacromolecules. 2009;10(10):2954-959.

27. Liu X, Ma L, Mao Z, et al. Chitosan-based biomaterials for tissue repair and regeneration. Adv Polym Sci. 2011;244:81-127. 
28. Eivazzadeh-Keihan R, Bahojb Noruzi E, Khanmohammadi Chenab K, et al. Metal-based nanoparticles for bone tissue engineering. J Tissue Eng Regen Med. 2020;14(12):1687-714.

29. Gherasim O, Puiu RA, Bîrcă AC, et al. An updated review on silver nanoparticles in biomedicine. Nanomaterials(Basel). 2020;10(11):2318.

30. Patil S, Singh N. Antibacterial silk fibroin scaffolds with green synthesized silver nanoparticles for osteoblast proliferation and human mesenchymal stem cell differentiation. Colloids Surf B Biointerfaces. 2019;176:150-55.

31. Niu X, Wei Y, Liu Q, et al. Silver-loaded microspheres reinforced chitosan scaffolds for skin tissue engineering. European Polymer Journal. 2020;134:109861.

32. Li DS, Yuan YH, Tu HJ, et al. A protocol for islet isolation from mouse pancreas. Nat Protoc. 2009;4(11):1649-652.

33. Kumar M, Nandi SK, Kaplan DL, et al. Localized immunomodulatory silk macrocapsules for islet-like spheroid formation and sustained insulin production. ACS Biomater Sci Eng. 2017;3(10):2443-456.

34. Shiroi A, Yoshikawa M, Yokota H, Fukui H, Ishizaka S, Tatsumi K, Takahashi Y. Identification of insulinproducing cells derived from embryonic stem cells by zinc-chelating dithizone. Stem cells. 2002;20(4):28492.

35. Douros JD, Lewis AG, Smith EP, et al. Enhanced glucose control following vertical sleeve gastrectomy does not require a $\beta$-cell glucagon-like peptide 1 receptor. Diabetes. 2018;67(8):1504-511.

36. Bai C, Gao Y, Li Q, et al. Differentiation of chicken umbilical cord mesenchymal stem cells into beta-like pancreatic islet cells. Artif Cells Nanomed Biotechnol. 2015;43(2):106-11.

37. Khorsandi L, Nejad-Dehbashi F, Ahangarpour A, et al. Three-dimensional differentiation of bone marrowderived mesenchymal stem cells into insulin-producing cells. Tissue Cell. 2015;47(1):66-72.

38. Jalili RB, Ghahary A. Biocompatible scaffolds: An effective means for improving islet cells viability and function. Int J Diabetes Clin Res. 2017;4(2):1-5.

39. Brereton MF, Vergari E, Zhang Q, Clark A. Alpha-, delta- and PP-cells: are they the architectural cornerstones of islet structure and co-ordination? J Histochem Cytochem. 2015;63(8):575-91.

40. Bhat S, Tripathi A, Kumar A. Supermacroprous chitosan-agarose-gelatin cryogels: in vitro characterization and in vivo assessment for cartilage tissue engineering. J R Soc Interface. 2011;8(57):540-54.

41. Tripathi A, Melo JS. Preparation of a sponge-like biocomposite agarose-chitosan scaffold with primary hepatocytes for establishing an in vitro 3D liver tissue model. RSC Adv. 2015;5(39):30701-710.

42. Kim JW, Vang S, Luo J, et al. Human islet co-cultured with bone marrow mesenchymal stem cells in 3D scaffolding may augment pancreatic beta cell function. J Biomater Tissue Eng. 2017;7(3):203-09. 